\title{
CARCINOSARCOMA DE ENDOMETRIO
}

\author{
INSTITUTO NACIONAL DE CANCEROLOGIA.
}

\section{Dr. Enrique Leaño P.*}

Preferiblemente llamado tumor Mülleriano mixto maligno. Es un tumor raro de endometrio y todavía más escaso de la mucosa cervical. Originado en el estroma y con varios elementos sarcomatosos de origen mesenquinal y elementos carcinomatosos de potencial mülleriano.

Wellis, Shapir y Vass (1) dudan de la validez de estos tumores como entidad biológica. Muchos de los tumores descritos en la literatura, uterinos o de otros órganos, han sido interpretados por estos autores como representaciones pleomórficas de carcinomas primarios.

\section{Caracteres clínicos}

Generalmente afecta a mujeres de mediana edad, en especial en la quinta década de la vida. El tumor puede localizarse en cualquier parte de útero, aunque es más frecuente en el cuerpo.

La sintomatología no es característica.

El diagnóstico suele obtenerse después de la intervención quirúrgica al efectuar el examen anatomopatológico.

En ocasiones falta el síntoma hemorrágico por completo. Este es de importancia cuando ocurre durante la menopausia y hay coincidencia con leiomiomas. En las mujeres jóvenes pueden presentarse menstruaciones abundantes, sangrados intermenstruales, o ambos a la vez. A veces hay masas pelvianas de considerable volumen, sin anormalidades menstruales. Lo mismo que en el carcinoma puede haber secreción anormal en las fases tempranas; suele ser fluída, acuosa y finalmente sanguinolenta. Cuando hay necrosis y ulceración se vuelve fétida y puede contener fragmentos de tejido.

El crecimiento rápido de tumores miomatosos coincidiendo con hemorragia, debe sugerirnos la posibilidad de esta lesión.

El dolor puede presentarse como síntoma, aunque regularmente no hace su aparición hasta las fases infiltrativas tardías de la enfermedad, en las cuales es intenso y continuo. En estados avanzados hay anemia y caquexia.

La recidiva y las metástasis seguidas de muerte suelen ser rápidas. La extensión se realiza por continuidad directa, por vía sanguínea o linfática. Los órganos más atacados son hígado y pulmones. La mayoría de las \footnotetext{
ogía.
} 
pacientes viven menos de dos años y presentan recurrencia pélvica local. El carcinosarcoma puede dar metástasis como carcinoma, sarcoma, o combinación de ambos.

Stout (2) ha visto muchos tumores dando Metástasis como carcinomas o sarcomas solamente, algunas veces como carcinoma en unos ganglios y sarcoma en otros. Otros autores han visto tumores dando metástasis como carcinosarcoma o carcinoma, pero nó como sarcoma puro.

\section{Anatomía Patológica}

Aspecto macroscópico: el útero puede encontrarse aumentado de tamaño de dos a tres veces su tamaño normal. Casi siempre está lleno y distendido por numerosas masas polipoides adheridas por una base ancha al cuerpo. Son blandas friables y de coloración blanca, gris o roja.

Las superficies de corte son homogéneas, con pequeños quistes, algunas veces con necrosis $y$ hemorragia, $y$ ocasionalmente, con evidencia macroscópica de invasión miometrial. El endometrio generalmente es pálido y delgado.

Aspecto microscópico: las masas polipoides están constituídas por una mezcla de elementos carcinomatosos y sarcomatosos con varios grados de diferenciación. Los elementos carcinomatosos son generalmente adenocarcinoma y ocasionalmente carcinoma escamo-celular.

La falta de diferenciación puede dificultar el diagnóstico, cuyo tipo histológico imposibilita la identificación del tejido.

Con frecuencia hay tendencia definida hacia la formación de vasos con paredes del gadas y áreas de hemorragia y necrosis.
En la mayoría de los casos hay evidencia histológica de invasión miometrial.

Meyer (3) ha explicado esta entidad morfológica en tres posibilidades:

1) Tumores de colisión: dos tumares de desarrollo independiente se invaden el uno al otro.

2 ) Tumores de combinación: resultan de dos elementos blastomatosos diferentes que se derivan de una célula primitiva.

3 ) Tumores de composición: cuando el parénquima y el estroma se han hecho blastomatosos.

De acuerdo con Stout, el tumor de colisión es la contigüidad accidental de dos tumores independientes. Fuede haber invasión de un tumor al otro en unos pocos milímetros y generalmente el carcinoma es el invasor.

\section{Material}

Siete casos de carcinosarcoma de endometrio del Instituto Nacional de Cancerología, el primero en 1958 y el último en 1970, se revisan.

\section{CASO 1}

HISTORIA CLINICA № 45.284 .

Edad: 70 años.

Lugar de nacimiento: Túquerres (Nariño).

Procedencia : Pasto.

Antecedentes Ginecológicos: Menarquia $13 \mathrm{ci}$ clos $28 \times 4-5$. Menopausia desde hace 12 años.

Paridad: GO PO AO.

Motivo de consulta: Sangrado genital de dos meses de evolución.

Lesión encontrada: Tejido friable en la cavidad uterina.

Estudio A.P. Carcinosarcoma de endometrio. 
Tratamiento inicial: Radium intrauterino e intravaginal. Intrauterino 7.000 mgrs, hora. Precervical 3.200 mgrs. hora.

Tratamiento complementario: No hubo.

\section{CASO 2}

HISTORIA CLINICA No 69.480 .

Edad: 40 años.

Lugar de nacimiento: Chiscas (Boyacá).

Procedencia: Bogotá.

Antecedentes Ginecológicos: Menarquia 12 ciclos $30 \times 4$.

Paridad: G1 PI AO.

Motivo de consulta: Sangrado genital con 4 años de evolución. Tumor abdominal de crecimiento rápido.

Síntomas: Dolor hipogástrico.

Lesión encontrada: Masa tumoral que ocupa fosa ilíaca derecha, flanco derecho, hipogastrio y parte de fosa izquierda. De consistencia dura, doloroso y fijo.

Citología: Pap. V.

Estudio A.P. Carcinosarcoma de endometrio.

Tratamiento inicial: Histerectomía total con salpingooforectomía bilateral.

Tratamiento complementario: No terminó tratamiento con $R X$ profundos que había iniciado.

Evolución: Muere con peritonitis, obstrucción $y$ trombosis de la vena mesentérica.

\section{CASO 3}

HISTORIA CLINICA No 79.639.

Edad: 55 años.

Lugar de nacimiento: Bogotá.

Procedencia: Bogotá.

Antecedentes Ginecológicos: Menarquia $14 \mathrm{ci}$ clos $28 \times 3$. Menopausia desde hace 3 años.

Paridad: G10 P7 A3.

Motivo de consulta: Secreción genital sanguinolenta con 2 años de evolución.

Lesión encontrada: Masa polipoide que reemplaza el cérvix.

Citología: Pap. II.
Estudio A.P.: Carcinosarcoma de endometrio.

Tratamiento inicial: Histerolinfadenectomía radical.

Tratamiento complementario: Dosis total de cobalto y radium $10.056 \mathrm{r}$ en punto $\mathrm{A}$. 6.490 $r$ en punto $B$.

Evolución: Metástasis pulmonares y pélvicas a los 7 meses.

La paciente se pierde de vista.

CASO 4.

HISTORIA CLINICA No 87.394

Edad: 60 años.

Lugar de nacimiento: Toca (Boyacá).

Procedencia: Toca (Boyacá).

Antecedentes Ginecológicos: Menarquia $15 \mathrm{ci}$ clos $30 \times 3$. Menopausia a los 58 años.

Paridad: G3 P3 AO.

Motivo de Consulta: Sangrado genital de 4 meses de evolución.

Síntomas: Dolor hipogástrico, vómito postprandial.

Lesión encontrada: Masa tumoral que ocupa toda la pelvis.

Citología: Pap. II. trio.

Estudio A.P.: Carcinosarcoma de endomeTratamiento inicial: Histerolinfadenectomía radical. Thiotepa durante 5 días.

Evolución: Se hacen evidentes metástasis pulmonares en el $9^{\circ}$ día post-operatorio y muere a los 24 días por depresión medular.

\section{CASO 5}

HISTORIA CLINICA No 91.101.

Edad: 60 años.

Lugar de nacimiento: Timaná (Boyacá).

Procedencia: Pasca (Cundinamarca).

Antecedentes Ginecológicos: Menarquia 14 ciclos $30 \times 3$. Menopausia hace 20 años.

Paridad: GO PO AO.

Motivo de consulta: Sangrado genital de 5 meses de evolución. 
Lesión encontrada: Utero aumentado de tamaño al doble de lo normal.

Citología: Pap. II.

Estudio A.P. Carcinosarcoma de endometrio.

Tratamiento inicial: Histerectomía total con salpingooforectomía bilateral.

Evolución: Reproducción tumoral en la cúpula vaginal, metástasis pulmonar. Muere a los dos meses.

\section{CASO 6}

HISTORIA CLINICA No 103.819.

Edad: 56 años. ca).

Lugar de nacimiento: Quetame (Cundinamar-

\section{Procedencia: Acacías (Meta).}

Antecedentes Ginecológicos: Menarquia $15 \mathrm{ci}$ clos $30 \times 4$. Menopausia desde hace 9 años.

Paridad: G4 P4 AO.

Motivo de consulta: Secreción sanguinolenta por vagina y sangrado de 7 meses de evolución.

Lesión encontrada: Tumor vascularizado de $10 \mathrm{cms}$. $\times 18$ que protruía por vulva.

Tratamiento inicial: Extirpación del tumor.

Tratamiento complementario: Cobalto y radium: $5.591 \mathrm{r}$ en punto A. $4.900 \mathrm{r}$. en punto $\mathrm{B}$.

Estudio A.P.: Carcinosarcoma reproducido en vagina.

Evolución: Muere a los 5 meses.

\section{CASO 7}

HISTORIA CLINICA No 106.035.

Edad: 45 años.

Lugar de nacimiento: Duitama (Boyacá).

Procedencia: Duitama (Boyacá).

Antecedentes Ginecológicos: Menarquia 15 ciclos $28 \times 4$.
Paridad: G5 P5 AO.

Motivo de consulta: Sangrado genital con 2 años de evolución. Le practicaron histerectomía sub-total en otra Institución.

Informe A.P.: Carcinosarcoma de endometrio.

Lesión encontrada: Vagina infiltrada en su pared lateral izquierda. Invasión vesical.

Estudio histológico: Carcinosarcoma en pared vesical.

Tratamiento complementario: Radioterapia : cobalto $6.000 \mathrm{R}$ en toda la pelvis. Hasta Abril de 1972 se encontraba en buenas condiciones sin evidencia de evolución tumoral.

\section{Resumen y Conclusiones}

19) El Carcinosarcoma de Endometrio, se presentó en mujeres maycres de 40 años, la edad osciló entre 40 y 60 años.

2.) El síntoma frecuente fué el sangrado genital; cinco pacientes eran menopáusicas.

3) El tratamiento básico fue la Histerectomía total, complementada con radium y cobaltoterapia.

49) Los resultados fueron malos, debido a lesiones muy avanzadas.

5) La supervivencia muy pobre excepto en la última paciente.

\section{BIBLIOGRAFIA}

1 HERTING y CORE. Atlas of Tumor Patology. Aparato Genital Femenino. Tumors of the Female. Sex organs. Pág. 223. 1960.

2 NOVAK WOODRUFF: Ginecología y Obstetricia. Tratado Anatomopatológico Clínico y Endocrinológico. Pág. 243. 1964.

3 ARTHUR HERTIG: Tumors of the Female. Sex Organs. Armed forces Institute of $\mathrm{Pa}$ thology. Carcinosarcoma. P. 33 (II) 223. 